\title{
On the suitability of lanthanides as actinide analogs
}

Kenneth Raymond, and Geza Szigethy

Chemistry Department, University of California, Berkeley, CA, 94720-1460

and Lawrence Berkeley National Laboratory, Berkeley, CA 94720

\section{ABSTRACT}

With the current level of actinide materials used in civilian power generation and the need for safe and efficient methods for the chemical separation of these species from their daughter products and for long-term storage requirements, a detailed understanding of actinide chemistry is of great importance. Due to the unique bonding properties of the $f$-elements, the lanthanides are commonly used as structural and chemical models for the actinides, but differences in the bonding between these $4 \mathrm{f}$ and $5 \mathrm{f}$ elements has become a question of immediate applicability to separations technology. This brief overview of actinide coordination chemistry in the Raymond group at UC Berkeley/LBNL examines the validity of using lanthanide analogs as structural models for the actinides, with particular attention paid to single crystal X-ray diffraction structures. Although lanthanides are commonly accepted as reasonable analogs for the actinides, these comparisons suggest the careful study of actinide materials independent of their lanthanide analogs to be of utmost importance to present and future efforts in nuclear industries.

\section{INTRODUCTION}

The current primary use of actinide elements globally is as fissile material for civilian power plants, an industry that generates tons of spent nuclear material annually. Undoubtedly, the benefits of utilizing a carbon-free power source in an ever-industrializing world are clearer in the light of the uncertainty of global oil resources and the growing awareness of the effect of greenhouse gasses on the global climate. However, the inherent dangers associated with the actinide waste materials from this industry and their proper treatment procedures has been an area of intense interest and heated debate in both the scientific community and general public. While some countries such as France have employed well-established solution chemistry extractions such as the PUREX process to separate the fission daughters from actinide material in spent fuel, countries such as the United States decide instead to store their radioactive waste materials in geological repositories to avoid the proliferation of pure transuranium elements that can be attained cleanly from many solution separation techniques. ${ }^{1}$

The projected necessary lifetime of geological repository containment integrity is on the order of 10,000 years, making the leaching of nuclear material into the environment and subsequent human exposure to radioactive material a significant safety concern. ${ }^{2}$ In order to anticipate the chemical behavior of actinide-bearing materials for so long a lifetime the detailed chemistry of actinide elements must be understood in great detail so that proper storage materials are used to avoid the potential for radiation leaks. In case there is exposure of the environment to these materials, however, as well as to address already-existing clean up efforts, there must also exist well-understood protocols for the removal of actinides from contaminated systems, whether environmental, inorganic, or biological in nature. 
Since the discovery of fission and the synthesis of transuranium actinides in the mid $20^{\text {th }}$ century, there has been a great deal of development in actinide solution chemistry as it applies to extraction processes, improvement and elaboration upon which continue today. ${ }^{3}$ However, as has been emphasized by our research group at the University of California at Berkeley and Lawrence Berkeley National Laboratory, there is still much to be explored in actinide chemistry, specifically in the structural properties and coordination chemistry of actinides in their various oxidation states.

Actinide elements, like their lanthanide $f$-block partners, typically express bonding behavior that is primarily electrostatic in nature, exhibiting little geometric preferences, in stark contrast to the transition metals whose bonding characteristics and geometries are strongly influenced by valence $d$-orbitals. Along with our collaborators in actinide sciences ranging from biology, physical chemistry, and solution state thermodynamics, we have been studying the interaction of actinide elements with ligands inspired by siderophores, which are low-molecular weight ligands produced by bacteria which utilize bidentate, all-oxygen donating moieties, some analogs of which are illustrated in Figure 1. ${ }^{4}$

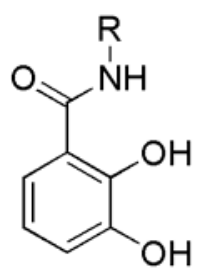

(a)

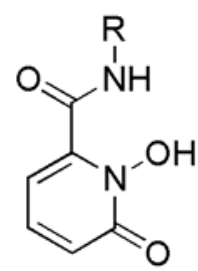

(b)<smiles>[R]NC(=O)c1ccn(C)c(=O)c1O</smiles>

(c)

Figure 1. Siderophore-type binding moieties utilized by the Raymond group and collaborators: (a) catechol amide (CAM); (b) 1,2-hydroxypyridinone amide (1,2-HOPO); (c) Me-3,2hydroxypyridinone amide (Me-3,2-HOPO).

Because there are few examples of transition metals displaying the cation radius and coordination modes observed in the actinides, the lanthanides are typically used as structural analogs to provide non-radioactive actinide models. The large variety of ionic radii in the lanthanide series caused by the lanthanide contraction provide structural models for actinide cations in various oxidation states, a fact we commonly utilize in our own work in preliminary studies of actinide bonding. However, subtle differences observed in our comparisons between lanthanide and actinide structures indicate that the use of lanthanide analogs may not always be appropriate. In fact, the recognition and exploitation of slight chemical differences between the lanthanides and actinides has become of utmost importance as the need to separate and store actinides safely relies upon our ability to distinguish actinides from all other elements, including the lanthanides.

\section{DISCUSSION}

Research involving actinide elements must inevitably encounter the difficulties and challenges of working with radioactive materials, whether they be naturally-occurring actinides such as thorium and uranium, or man-made such as neptunium, plutonium and the other transuranium elements. For this reason, using lanthanides as actinide models is attractive, as it avoids the procedural challenges of handling radioactive species and utilizes $f$-block elements 
that in many ways resemble the actinides in chemistry and structure: the bonding in these species is typically considered ionic in nature, displaying little to no directional coordinative preference in their complexes. It is commonly accepted that this is due to the contraction of the valence orbitals as compared to transition metals in which the $d$-orbitals are largely responsible for the geometric and electronic behavior of coordination and organometallic species. Thus, the lanthanides are by elimination the only reasonable candidates for use as actinide analogs, as there are no other elements of similar electronic configuration and coordination mode in the periodic table. However, among the large variety of actinide fission products are several lanthanides, and removal of these elements from the actinides in spent fuel has is a challenge due to the similar bonding nature of the $f$-block elements, and thus the properties that make lanthanides convenient actinide surrogates in structural modeling makes them more a nuisance than a boon for the actinide and separations chemist. Because of this, a more detailed examination of actinide chemistry is needed to determine exactly how these differences can manifest themselves and in what ways they can be exploited. ${ }^{5}$

\section{Chemical behavior}

The bonding in $f$-block metals at a cursory level is often interpreted as a primarily ionic phenomenon, with bond distances and coordination geometries determined simply by ionic radii and ligand-ligand repulsion. This view treats the electronic configuration of the $f$-electrons as relatively unimportant, assuming that the $f$-orbitals do not exhibit the necessary radial extension and directionality to significantly influence bonding behavior. However, since the $5 f$ orbitals have greater radial extension than the $4 f$ orbitals, the question of the level of covalency in actinide elements has been an area of continued debate and investigation. ${ }^{6}$ Elucidating the nature of the effect these orbital properties have on the relative amounts of covalency found in $f$-block elements has been an ongoing effort in actinide science, and recent theoretical and experimental work on bond distances between actinides and lanthanides of similar ionic radii with soft donor ligands support the presence of increased covalency in the actinides. ${ }^{7}$,

Additionally, there is a more obvious difference between the two rows of $f$-elements that attests to the difference between the rows, namely the rich redox chemistry of the early actinides (easily accessible oxidation states ranging from III to VI), whereas the chemistry of the lanthanides is primarily dominated by the $3+$ oxidation state. This oxidative variability in the actinides has been explained by the close spacing of the $d$ and $f$ levels in these elements, and attests to a slight, yet significant electronic difference between the two $f$-element periods. A result of this richer redox chemistry is the propensity of early actinides to adopt high-valent dioxo cation species which are strictly linear species, for which there are no chemical and structural analogs anywhere in the periodic table outside of the actinides. Thus, the chemistry of the actinides even at first glance is more varied than the lanthanides, making their use as analogs require a judicious choice of element and circumstances so these redox and subsequent structural differences caused by the anisotropy of the linear dioxo cations do not make structural analogies meaningless.

\section{Coordination chemistry}

While the observations above are of the general literature on actinides, our group along with our collaborators is active in the investigation of a physical manifestation of subtle $f$ - 
element electronic configuration consequences, namely the coordination geometry in actinide complexes. In our work using siderophore-inspired ligands we employ lanthanide elements of similar charge and ionic radius as structural models before pursuing similar results with actinides. Typically, the coordination geometries adopted by the lanthanide and actinide complexes can be likened to a coordination polyhedron as described by the Kepert model of electrostatic repulsion of points on a sphere, with minor allowances made for the size and coordination mode of the ligands. ${ }^{8}$ However, it is the deviations from this predicted electrostatic behavior that are most interesting to us in our investigations.

More specifically, we utilize $\mathrm{Ce}(\mathrm{IV})$ as a structural analog for $\mathrm{Pu}(\mathrm{IV})$, which is a widelyaccepted practice as these species have identical charges and ionic radii. In general, $\mathrm{Ce}(\mathrm{IV})$ has proven to be a reasonable analog for $\mathrm{Pu}(\mathrm{IV})$ with these ligands, but there exist minor M-L bond distance differences between the lanthanide and actinide structures (Table I): while ligands containing HOPO coordination moieties display very similar bond distances between $\mathrm{Ce}(\mathrm{IV})$ and $\mathrm{Pu}(\mathrm{IV})$ species, complexes with maltol ligands (Figure 3) display statistically different bond lengths. Although these discrepancies are slight and as yet incompletely explained, they contribute to the observable differences between lanthanide and actinide coordination behavior that is becoming important in the field of radionuclide separations and sequestration.

Table I. Observed M-O bond distances in previously-reported crystal structures of $\mathrm{Ce}(\mathrm{IV})$ and $\mathrm{Pu}(\mathrm{IV})$ complexes of type $\mathrm{ML}_{\mathrm{n}}$ using siderophore-type binding moieties. Average values are given when more than one unique $\mathrm{M}-\mathrm{O}$ bond exists in the crystal structure. ${ }^{9,10}$

\begin{tabular}{cccc} 
Ligand & M(IV) & $\begin{array}{c}\text { M-O } \\
\text { (phenoxide/N- } \\
\text { oxide), } \AA\end{array}$ & $\begin{array}{c}\text { M-O } \\
\text { (carbonyl), } \AA\end{array}$ \\
\hline \multirow{2}{*}{ 1,2-HOPO } & $\mathbf{C e}$ & $2.33(2)$ & $2.35(3)$ \\
& Pu & $2.32(2)$ & $2.31(2)$ \\
\hline \multirow{2}{*}{ 5LiO-Me-3,2-HOPO } & $\mathbf{C e}$ & $2.30(1)$ & $2.41(2)$ \\
& $\mathbf{P u}$ & $2.28(3)$ & $2.38(3)$ \\
\hline \multirow{2}{*}{ Maltol } & $\mathbf{C e}$ & $2.276(3)$ & $2.441(3)$ \\
& $\mathbf{P u}$ & $2.286(3)$ & $2.419(3)$ \\
\hline \multirow{2}{*}{ BrMaltol } & $\mathbf{C e}$ & $2.245(3)$ & $2.503(3)$ \\
& $\mathbf{P u}$ & $2.24(3)$ & $2.36(3)$ \\
\hline
\end{tabular}<smiles>Cc1occ(Br)c(=O)c1O</smiles>

Figure 3. Maltol (left) and bromomaltol (BrMaltol, right). ${ }^{11}$

Because the identification of coordination polyhedra of $f$-block elements (as described by Kepert $)^{8}$ is more difficult to discriminate than with transition metal species, we have for some time employed a shape measure to determine which coordination geometry is most closely approached in the crystal structures of eight-coordinate coordination compounds. ${ }^{10}$ In eightcoordinate complexes the idealized possible coordination polyhedra are the square antiprism, bicapped trigonal prism, and trigonal-faced dodecahedron which have $D_{4 d}, C_{2 v}$, and $D_{2 d}$ coordination geometries, respectively. As Table II indicates, the shape measure values for the 
$\mathrm{Ce}(\mathrm{IV})$ and $\mathrm{Pu}(\mathrm{IV})$ complexes are in many cases similar, but with 1,2-HOPO ligands appears to lie near an energetic cusp between $C_{2 v}$ and $D_{2 d}$ geometries, suggesting that the coordination environment could be significantly affected by relatively small changes in ligand geometry. Additionally, the maltol ligands exhibit a coordination behavior that seems to prefer a $D_{2 d}$ coordination geometry, but what is not revealed by the shape measure analysis is that in the case of the $\mathrm{Ce}(\mathrm{BrMaltol})_{4}$ structure, a new and previously unknown ligand arrangement is adopted by the complex while not adopted by the analogous $\mathrm{Pu}(\mathrm{IV})$ complex.

Table II. Shape measure values calculated from crystal structures of $\mathrm{ML}_{\mathrm{n}}$ complexes of $\mathrm{Ce}(\mathrm{IV})$ and $\mathrm{Pu}(\mathrm{IV})$ using siderophore-type binding moieties. Bold and italic values indicate the minimum shape measure for the complex and therefore the most appropriate description of the coordination geometry., ${ }^{9,10}$

\begin{tabular}{ccccc} 
Ligand & $\mathbf{M}(\mathbf{I V})$ & $\boldsymbol{D}_{4 d}$ & $\boldsymbol{C}_{2 v}$ & \multicolumn{1}{c}{$\boldsymbol{D}_{2 \boldsymbol{d}}$} \\
\hline \multirow{3}{*}{ 1,2-HOPO } & \multirow{2}{*}{$\mathrm{Ce}$} & 16.0470 & 13.5587 & $\mathbf{1 3 . 3 9 0 4}$ \\
& & 18.9709 & 13.1182 & $\mathbf{9 . 9 1 0 4}$ \\
\cline { 2 - 5 } & \multirow{2}{*}{$\mathbf{P u}$} & 16.3136 & $\mathbf{1 0 . 0 0 6 3}$ & 11.4562 \\
& & 18.7766 & 13.8437 & $\mathbf{9 . 3 7 8 1}$ \\
\hline \multirow{2}{*}{ 5LiO-Me-3,2-HOPO } & $\mathrm{Ce}$ & 12.1749 & $\mathbf{8 . 7 7 9 9}$ & 9.5145 \\
& \multirow{2}{*}{$\mathbf{P u}$} & $\mathbf{6 . 4 8 6 2}$ & 10.4150 & 13.4384 \\
\hline \multirow{2}{*}{ Maltol } & $\mathbf{9 . 3 7 8 0}$ & 11.9781 & 14.2430 \\
& & $\mathbf{1 0 . 0 6 1 6}$ & 10.7068 & 12.2529 \\
\hline \multirow{2}{*}{ BrMaltol } & $\mathbf{P u}$ & 15.1909 & 13.2243 & $\mathbf{3 . 6 6 7 6}$ \\
& $\mathbf{C e}$ & 15.0645 & 13.3768 & $\mathbf{3 . 3 7 4 0}$ \\
\hline & $\mathbf{P u}$ & 16.1235 & 12.6109 & $\mathbf{3 . 8 4 6 5}$ \\
\hline
\end{tabular}

It may seem that these differences are random and dictated only by the ligands under investigation rather than the electronics of the metal center. However, while the expanded $d$ - and $f$-orbitals may contribute only a small amount of covalency to the bonding behavior of actinides, this potentially small value cannot be ignored in light of the small energetic differences between coordination polyhedra as illustrated by the theoretical studies of Hay and coworkers on the calculated structures of U(IV)-catecholate complexes. ${ }^{12}$ It is exactly the small differences between the lanthanide and actinide geometries and coordination preferences that attest to the need for actinide chemistry to focus on the use of actinides independent from their lanthanide analogs because these small differences will provide the basis of next-generation extraction technology.

\section{CONCLUSIONS}

Using lanthanides as actinide models has many advantages associated with the manipulation of non-radioactive materials. Utilizing lanthanides as actinide analogs is admittedly a necessity in $f$-block chemistry, as no other class of elements more closely approaches the chemical and geometric behavior of the actinides than do the lanthanides, and these similarities are constantly being utilized in the expansion of actinide science and $f$-element chemistry in general. However, a cursory examination of the simple electronic behavioral differences as well as some compiled coordination behavior analysis reveals some inconsistencies between Ln and 
An species that suggest the presence of small, yet potentially significant differences in their chemical behavior, the intricacies of which are being explored by us as well as numerous actinide chemists the world over.

Although the differences are not fully understood at this time, the observable differences indicate the importance of recognizing that lanthanide models of the actinides are approximate. and may be misleading; hence significant effort should continue to be invested in the exploration of how the lanthanides and actinides differ, rather than how they are similar. It is these differences that will have significant consequences on how the world as a whole addresses both legacy and future actinide materials.

\section{ACKNOWLEDGMENTS}

This work was supported by the Director, Office of Science, Office of Basic Energy Sciences, Chemical Sciences, Geosciences, and Biosciences Division of the U.S. Department of Energy under Contract Number DE-AC02-05CH11231 at LBNL.

\section{REFERENCES}

1 Warren I. Finch, U.S. Geological Survey Bulletin 2179-A (2003).

2 G. S. Bodvarsson, W. Boyle, R. Patterson et al., Journal of Contaminant Hydrology 38, 3 (1999).

$3 \quad$ A. P. Paiva and P. Malik, J. Rad. Nuc. Chem. 261 (2), 485 (2004).

4 A. E. V. Gorden, J. Xu, and K.N. Raymond, Chem. Rev. 103, 4207 (2003).

5 N.N. Greenwood and A. Earnshaw, Chemistry of the Elements. (Pergamon Press, New York, 1997).

$6 \quad$ G. Schreckenback, P. J. Hay, and R. L. Martin, J. Comp. Chem. 20, 70 (1999).

7 A. J. Gaunt, S. D. Reilly, A. E. Enriquiez et al., Inorg. Chem. 47, 29 (2008); M. Roger, L. Belkhiri, T. Arliguie et al., Organometallics 27, 33 (2008).

8 D.L. Kepert, Inorganic Stereochemistry. (Springer-Verlag, New York, 1982).

9 A. E. V. Gorden, D. K. Shuh, B. E. F. Tiedemann et al., Chem. Eur. J. 11, 2842 (2005), 13, 378 (2007); A. E. V. Gorden, J. Xu, G. Szigethy et al., J. Am. Chem. Soc. 129 (21), 6674 (2007); G. Szigethy, J. Xu, A. E. V. Gorden et al., Eur. J. Inorg. Chem. Published online 28 Mar (2008).

10 J. Xu, E. Radkov, M. Ziegler et al., Inorg. Chem. 39, 4156 (2000).

11 J. H. Looker, R. J. Prokop, W. E. Serbousek et al., J. Org. Chem. 44 (19), 3408 (1979).

12 B. P. Hay, J. Uddin, and T. K. Firman, Polyhedron 23, 145 (2004). 